\title{
Tratamiento de la alergia a hongos
}

\section{Treatment of allergy to mushrooms}

\section{M.T. Lizaso, B.E. García, B. Gómez, A. Zabalegui, M.J. Rodríguez, A.I. Tabar}

\section{RESUMEN}

El tratamiento de los enfermos con alergia respiratoria se basa en medidas de control ambiental, tratamiento farmacológico e inmunoterapia.

En nuestro medio, los hongos representan la tercera causa de enfermedad respiratoria alérgica, siendo principalmente el género Alternaria el más frecuentemente implicado. Sin embargo, debido a que presentan randes dificultades en el dianóstico y tratan trolados sobre inmunoterapia con extractos fúngicos.

Se realizó un ensayo clínico con un extracto adecuado, estandarizado biológicamente, para el diagnóstico y tratamiento de los enfermos alérgicos a Alternatia y tratamiternatia. En una segu ediante inmunoterapia, en preparación depot y en pauta convencional, que fue de $0,1 \mathrm{mg} / \mathrm{ml}$ de Alt a 1 . Esta dosis se estableció como dosis de mantenimiento en la siguiente fase (ensayo doble ciego controlado con placebo) en la cual se determinó la eficacia y seguridad de la inmunoterapia con ese extracto, administrado en inmunoterapia a la dosis de mantenimiento referida, 28 pacientes con rinitis y/o asma por aleria a Altera 28 pacientes con initis y/o asma por alergia a Alternaria. Todos los pacientes alcanzaron la dosis preestablecida de mantenimiento de 1670 UBE. El tratamiento resultó eficaz, produciéndose una mejoría en los síntomas, función respiratoria, evaluación subjetiva de paciente y del médico y severidad de la enfermedad. La respuesta inmunológica apoyó la eficacia clínica, con un aumento de la IgG y un descenso de la IgE a lo largo del estudio. La tolerancia del tratamiento fue excelente, registrándose únicamente dos reacciones sistém, revistrand 711 dosis (0,28\% reac(radas).

Palabras clave. Alergia. Alternaria. Hongos. Inmunoterapia.

An. sis. sanit. Navar. 2003; 26 (Supl. 2): 129-137.

Este ensayo clínico ha sido subvencionado por el Departamento de Salud del Gobierno de Navarra en 1998.

\section{ABSTRACT}

The treatment of patients with respiratory allergy is based on environmental control measures, pharmacological and immunotherapy treatment.

The third cause of allergic respiratory disease in our environment is mushrooms, the most frequently involved being the Alternaria class. However, due to the great difficulties in their diagnosis and specific treatment, there are few controlled studies on immunotherapy with mushroom extracts.

A clinical test was carried out with a suitable biologically standardized extract for the diagnosis an treatment of patients allergic to Alternaria. A second phase determined the maximum tolerated dosage of his extract administered through immunotherapy, in depot preparation and in conventional dosage, which was $0,1 \mathrm{mg}$ /m olt a I This dosa was $0,1 \mathrm{mg} / \mathrm{ml}$ of Alt a l. This dosage was established a the maintenance dosage in the following phase (double blind test controlled with placebo), in which th efficacy and safety of the immunotherapy with this extract was determined, administered in mmunotherapy to the mentioned maintenance dosage, to 28 patients with rhinitis and/or asthma due to allergy to Alternaria. All the patients reached the preestablished maintenance dosage of 1670 BSU. The treatment proved efficient, producing an improvement treatment proved efficient, producing an inprovement in the symptoms, respiratory function, subjective evaluation of patient and doctor, and severity of the disease. The immunological response supported the clinical efficacy, with an increase in the IgG and a fall in the IgE over the course of the study. Tolerance to the treatment was excellent, with only two light systemic reactions registered in the 711 dosages administered ( $0.28 \%$ reactions/dosages administered).

Key words. Allergy. Alternaria. Mushrooms Immunotherapy.

\section{Correspondencia:}

$\mathrm{M}^{\mathrm{a}}$ Teresa Lizaso Bacaicoa

Sección de Alergología

Plaza de la Paz s/n

31002 Pamplona

Tfno: 948429308

FAX: 948429271

mlizasob@cfnavarra.es 


\section{INTRODUCCIÓN}

Los hongos pueden ser causantes de patología alérgica respiratoria, tanto de vías aéreas superiores como inferiores, en personas sensibles.

Los principales géneros de hongos causantes de alergia son Alternaria, Aspergi llus, Cladosporium y Penicillium, siendo Cladosporium en el norte de Europa y Alternaria en el área mediterránea y sobre todo en niños, los más frecuentemente implica$\operatorname{dos}^{1}$.

En trabajos recientes se ha comprobado que la prevalencia de alergia a hongos es mayor de lo que hasta ahora se pensaba y que los hongos, como causantes de asma bronquial y otras enfermedades del sistema respiratorio, han sido subestimados ${ }^{1}$ Las esporas fúngicas se encuentran en el aire a concentraciones muy superiores a las de los pólenes y en muchos casos son más pequeñas que los granos de polen pudiendo alcanzar así más fácilmente el tracto respiratorio inferior $\mathrm{y}$ producir asma $^{2}$. Aunque no se conoce exactamente la prevalencia de patología alérgica respiratoria por hongos y varía mucho según los diferentes autores y, sobre todo, en relación con la población analizada, según un estudio multicéntrico europeo promovido por el Subcomité de Aerobiología de la Academia Europea de Alergología en 1997 , un $9,5 \%$ de los pacientes con sospecha de alergia respiratoria estaba sensibilizado a Alternaria y/o Cladosporium, siendo España el país donde la prevalencia fue mayor (20\%) y Portugal donde fue menor $(3 \%)^{1}$. Actualmente se considera que los hongos son la tercera causa más frecuente de patología alérgica respiratoria, tras los ácaros y los pólenes. La mayoría de pacientes alérgicos a estos hongos presentan síntomas de modo perenne $\mathrm{e}^{2,3}$, aunque en nuestra zona, la máxima concentración de esporas de Alternaria en el ambiente y, por tanto, al menos en teoría, el pico de sintomatología en estos pacientes se produce durante los meses de verano.

A pesar de todo esto, dentro de los aeroalergenos, los hongos son los que han creado más dificultades para su estudio, lo que ha traído como resultado la falta de investigación y de trabajos publicados en este campo.

El diagnóstico de alergia a hongos puede ser difícil de realizar, probablemente porque los extractos comerciales disponibles hasta el momento para procedimientos diagnósticos no son muy eficaces, tendiendo a tener una baja actividad alergénica y gran variabilidad en su composición de lote a lote. La mayor parte de fracciones fúngicas inhaladas por los pacientes son esporas, aunque también se inhalan fragmentos de micelios. Con los hongos, a diferencia de con la mayoría de los restantes agentes etiológicos sensibilizantes, no está claro cuál es la fuente original sensibilizante, si los micelios, las esporas o sus metabolitos, por lo que no está claro cómo se deben producir los extractos con actividad antigénica ni como son los métodos más adecuados para su estandarización. Además, existe una gran variabilidad antigénica en las cepas fúngicas ${ }^{2,3}$. Por todo esto, la hipersensibilidad inmediata a hongos tiene importantes dificultades en el diagnóstico y aún más en el tratamiento específico mediante inmunoterapia.

\section{TRATAMIENTO}

El tratamiento de los enfermos con patología respiratoria producida por alergia a hongos, al igual que la causada por otros aeroalergenos, se basa en tres pilares fundamentales que se complementan medidas de control ambiental, farmacoterapia e inmunoterapia.

\section{Medidas de control ambiental}

La profilaxis encaminada a la disminución de la concentración de alergenos mediante medidas higiénicas sencillas de realizar, es la primera fase en el tratamiento de un paciente alérgico. Los hongos están ampliamente distribuidos en la naturaleza por lo que es muy difícil, si no imposible, su evitación por completo. Sin embargo hay un cierto número de precauciones que se pueden tomar para minimizar el contacto con ellos. Dado que el crecimiento de los hongos se ve favorecido por la humedad elevada, la oscuridad y el acumulo de polvo y mate- 
riales orgánicos ${ }^{2,3}$, las principales medidas ambientales a tener en cuenta son las siguientes ${ }^{4}$ :

En el exterior de las viviendas:

- Evitar acercarse a vegetación muerta o en estado de descomposición y no mover montones de hojas caídas en el suelo.

- Evitar tener vegetación densa cerca de la vivienda. Se deben eliminar las hojas muertas y evitar el acumulo de restos orgánicos cerca de la casa (basureros, estercoleros, etc.).

En el interior de la vivienda:

- Eliminar la humedad y airear las zonas oscuras y húmedas de la casa, utilizando en ellas pintura antimohos y fungicidas en sitios que tengan tendencia a humedecerse.

- Evitar plantas de interior y flores secas de adorno.

- La cocina y el cuarto de baño, por sus condiciones de humedad y calor, favorecen el desarrollo de hongos, por ello deben ser aireados y limpiados con soluciones antifúngicas (lejía).

- Evitar el almacenamiento de alimentos, sobre todo de frutas y verduras.

- No guardar nunca ropa o zapatos húmedos en un armario o en zonas de poca ventilación.

- Deshacerse lo antes posible de bolsas de basura que contengan restos de alimentos.

- Los aparatos de aire acondicionado y humidificadores favorecen el crecimiento y dispersión de los hongos por lo que si se tienen se deben limpiar con frecuencia y lavar los filtros con soluciones antifúngicas.

- Usar deshumificadores en épocas muy húmedas, pero teniendo cuidado de que tampoco se contaminen con hongos los circuitos del equipo.

- Limpiar las tuberías y desagües de la lavadora y del fregadero.

- Aspirar la habitación diariamente.

- Evitar que se acumule polvo en cualquier sitio.

\section{Farmacoterapia}

El tratamiento farmacológico es el segundo escalón en el tratamiento de la enfermedad alérgica. Los fármacos actualmente empleados en el tratamiento del asma se pueden dividir en fármacos antiinflamatorios (corticoides, cromonas y antileucotrienos) y broncodilatadores (agonistas $b_{2}$ ). En el caso de la rinitis los principales fármacos utilizados son antihistamínicos y corticoides nasales. La indicación de uno u otro de estos fármacos dependerá de la gravedad de la enfermedad, lo cual queda claramente detallado en las últimas guías publicadas para el manejo del asma y de la rinitis ${ }^{5,6}$.

\section{Inmunoterapia}

La inmunoterapia es hoy por hoy el único tratamiento etiológico de las enfermedades alérgicas respiratorias mediadas por anticuerpos IgE específicos. Su eficacia clínica ha sido demostrada siempre que se realice una indicación correcta, basada en la demostración de que la sensibilización al alergeno juega un papel relevante en el desarrollo de los síntomas y la severidad de la enfermedad, se disponga de extractos alergénicos de alta calidad adecuados para procedimientos terapéuticos, y se realice su administración a dosis apropiadas y durante un tiempo adecuado (3-5 años). Por otro lado, otro requisito previo a la instauración de la inmunoterapia debe ser la imposibilidad para evitar o eliminar al alergeno causante del cuadro clínico, como es el caso de los alergenos ubicuos, entre los que se encuentran los hongos. Así, actualmente, la inmunoterapia ha sido demostrada como un tratamiento eficaz de las enfermedades respiratorias alérgicas producidas por sensibilización a pólenes, ácaros del polvo doméstico, epitelios de animales y venenos de himenópteros, constatándose en múltiples estudios, que la inmunoterpia disminuye la severidad de la sintomatología y la necesidad de tratamiento farmacológico sintomático y demostrándose como el único tratamiento etiológico capaz de modificar el curso de la enfermedad respiratoria de causa alérgica ${ }^{7-10}$. La inmunoterpia consiste en la administración, generalmente subcutánea, de dosis progresivamente cre- 
cientes del material alergénico al que el paciente está sensibilizado. Existen varias pautas para la administración de la inmunoterpia, pero la más usada hasta ahora es la pauta convencional (aunque últimamente están tomando mucha fuerza las pautas agrupadas), en la que se administran dosis crecientes semanales en el periodo de iniciación, hasta alcanzar la dosis de mantenimiento, pasando posteriormente a una dosis mensual durante el periodo de continuación ${ }^{7}$.

Debido a las razones arriba indicadas acerca de la dificultad para realizar estudios sobre alergia a hongos y la escasa calidad de los extractos fúngicos disponibles en el mercado hasta el momento, tanto para fines diagnósticos como terapéuticos, existen muy pocos estudios controlados realizados sobre la eficacia y seguridad de la inmunoterpia con extractos fúngicos $^{11-18}$. Por ello, el Subcomité de Inmunoterapia de la Academia Europea de Alergología e Inmunología Clínica, ha señalado la necesidad de la realización de estudios controlados sobre inmunoterapia con extractos fúngicos estandarizados ${ }^{10}$.

Todo ello nos ha llevado a la realización de un ensayo clínico sobre inmunoterapia con un nuevo extracto de Alternaria estandarizado, con el fin de poder ofertar a los múltiples pacientes con patología respiratoria causada por alergia a hongos que diariamente diagnosticamos en nuestro servicio, un tratamiento etiológico eficaz y seguro con inmunoterapia, tal y como ya podemos ofrecer a otros pacientes con patología respiratoria alérgica causada por otros alergenos.

\section{ENSAYO CLÍNICO}

El estudio se compone de tres fases: la primera consiste en la obtención del extracto alergénico de alta calidad de $A$. alternata, estandarizado biológicamente y adecuado para el diagnóstico y tratamiento de pacientes alérgicos a este hongo; la segunda es la determinación de la dosis máxima tolerada de dicho extracto en inmunoterapia, y la última es el análisis de la eficacia y seguridad de la inmunoterapia mediante un ensayo doble ciego controlado con placebo.

\section{Fase 1 - Obtención de un extracto adecuado}

Objetivo: Obtener un extracto bien definido, caracterizado y reproducible de A. alternata, adecuado para el diagnóstico $\mathrm{y}$ tratamiento de los pacientes alérgicos a este hongo.

Material y métodos: los extractos alergénicos se obtuvieron del cultivo en un medio sintético de 4 cepas diferentes de $A$. alternata. A partir de este cultivo se prepararon extractos de 3 procedencias:

- Un extracto metabólico obtenido del filtrado del medio de cultivo.

- Un extracto periplasmático, de proteínas celulares de micelios/esporas sin romper.

- Un extracto somático, de proteínas celulares de micelios/esporas rotas.

La actividad alergénica de los 3 extractos fue evaluada biológicamente ${ }^{19-21}$ mediante titulación punto final en pruebas cutáneas en prick en 21 pacientes con rinitis y/o asma bronquial alérgico y monosensibilizados a A. alternata. Se utilizaron 4 concentraciones de cada extracto $(0,5 ; 1 ; 2$ y $5 \mathrm{mg} / \mathrm{ml}$ ) y las pruebas se realizaron por duplicado.

La caracterización inmunoquímica de los extractos se realizó mediante la determinación del contenido de alergeno mayor de $A$. alternata, Alt a 1 , por medio de un ELISA basado en anticuerpos monoclonales y mediante SDS-PAGE e "inmunoblotting".

Resultados: el extracto metabólico fue, con diferencia, el más reactivo de los tres con un valor de SPT, definido como la concentración del extracto que produce una pápula similar a histamina a $10 \mathrm{mg} / \mathrm{ml}$, de $2,09 \mathrm{mg} / \mathrm{ml}$. El SPT extrapolado, para el extracto periplasmático y somático fue de 28,84 y de $275,4 \mathrm{mg} / \mathrm{ml}$ respectivamente (Fig. 1).

También el extracto metabólico resultó ser el que contenía mayor cantidad de Alt a 1 , el $2,1 \%$ del total de proteínas del extracto, así como el que presentaba el patrón más amplio e intenso de unión de IgE en el SDS-PAGE y en el inmunoblotting. 


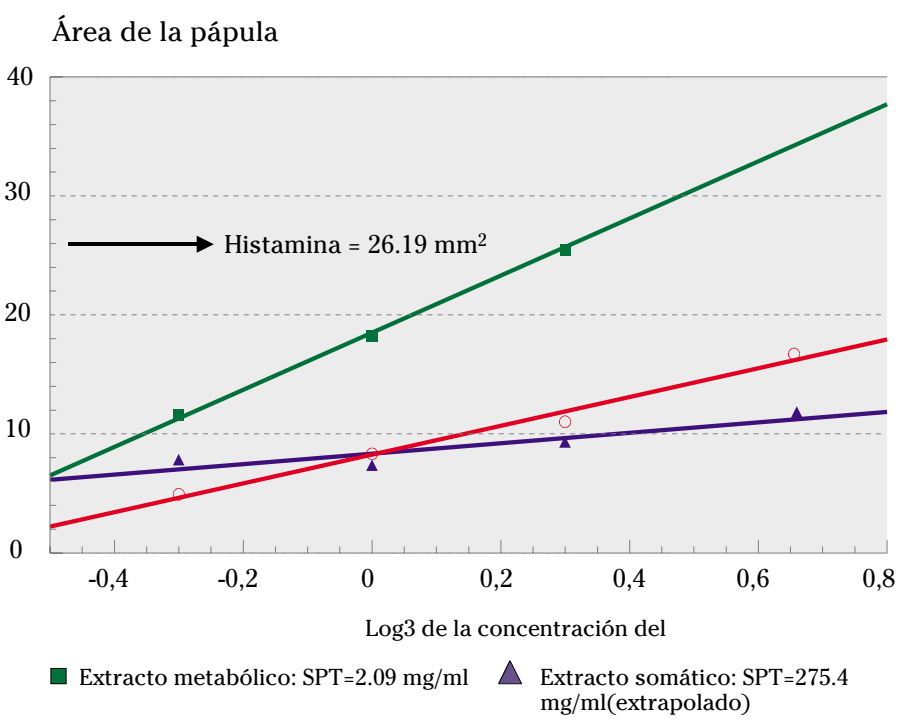

Figura 1. SPT de los tres extractos probados.

\section{Fase 2 - Determinación de la dosis máxima tolerada de inmunoterapia}

Objetivo: establecer la dosis máxima tolerada del extracto de Alternaria anteriormente obtenido para inmunoterapia.

Material y métodos: se seleccionaron 10 pacientes con rinitis y/o asma bronquial alérgico, monosensibilizados a Alternaria y con indicación de tratamiento inmunoterápico. A estos 10 pacientes se les administró inmunoterapia con el extracto anteriormente obtenido, en preparación depot y siguiendo una pauta convencional.

Se definió la dosis máxima tolerada de inmunoterapia como la mayor dosis tolerada por todos los pacientes (no reacciones sistémicas).

Se realizó un seguimiento posterior de estos pacientes, estableciendo 4 tiempos de estudio: T0 antes del inicio de inmunoterapia, T1 al alcanzar el mantenimiento, T3 al año de mantenimiento y T4 a los dos años de éste.

En todos los pacientes y en todos los tiempos de estudio se realizaron pruebas cutáneas en prick a 3 concentraciones progresivas, provocación conjuntival con salino y 3 concentraciones crecientes progre- sivas y se determinó la IgG e IgE específica a A. alternata.

Resultados: el $60 \%$ de los pacientes seleccionados eran varones y el $80 \%$ padecían asma. La edad media fue de 17,4 años.

La máxima dosis tolerada fue establecida en $1670 \mathrm{UBE}$, correspondiente a 0,167 mg del extracto liofilizado que contiene 0,1 mg de Alt a 1 (Fig. 2).

El área media de la pápula obtenida en prick a lo largo de los tiempos de estudio del seguimiento realizado a estos pacientes no mostró una tendencia clara.

La provocación conjuntival siguió una tendencia favorable, precisando concentraciones cada vez mayores para obtenerse una prueba positiva, e incluso siendo negativa en un paciente en T3 y en la mitad de los pacientes en T4.

La IgE específica a A. alternata fue disminuyendo de $\mathrm{T} 0$ a T3 y se mantuvo de $\mathrm{T} 3$ a T4. La IgG, por el contrario, presentó un aumento progresivo hasta T3, manteniéndose o disminuyendo de T3 a T4. En todos los casos los valores de IgE específica a $A$. alternata en T4 fueron muy inferiores a los registrados en $\mathrm{T} 0$ y por el contrario los de IgG específica fueron muy superiores al final del estudio respecto a su inicio. 


\section{№ Pacientes}

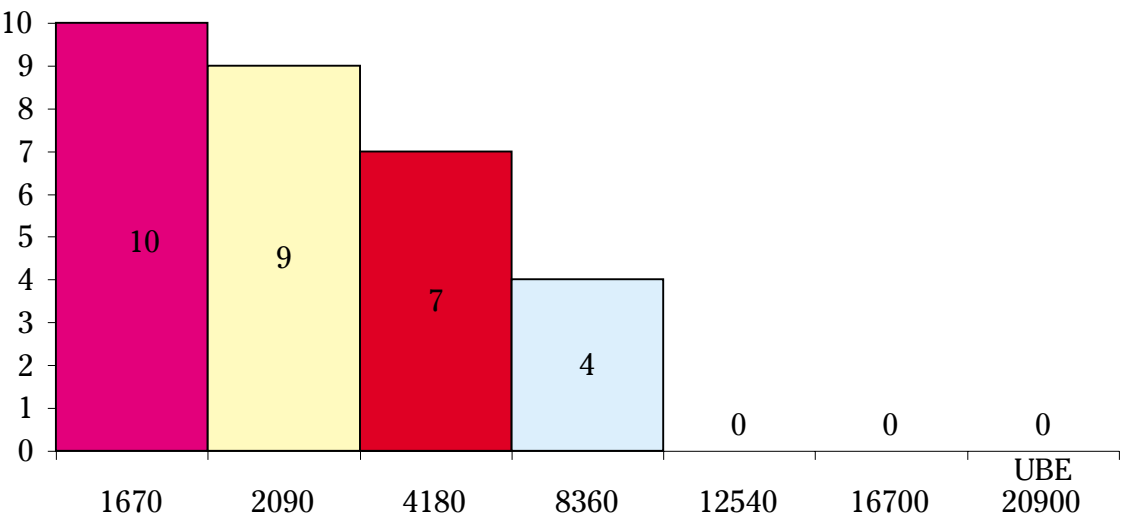

Figura 2. Dosis máxima tolerada.

\section{Fase 3 - Eficacia y seguridad de la inmunoterapia (Ensayo doble ciego)}

Objetivo: determinar la eficacia y seguridad de la inmunoterapia con el extracto de A. Alternata obtenido en la primera fase del estudio, estableciendo como dosis de mantenimiento la dosis máxima tolerada determinada en la fase anterior.

Material y métodos: se seleccionaron 28 pacientes con rinitis y/o asma bronquial monosensibilizados a Alternaria a los cuales se aleatorizó en dos grupos. A uno de ellos, el grupo activo, se le comenzó a administrar inmunoterapia con el extracto ya obtenido anteriormente, en preparación depot y siguiendo una pauta convencional. Al otro grupo, el placebo, se le administro únicamente excipiente o excipiente + histamina, siguiendo una pauta idéntica a la del grupo activo.

Se establecieron 4 tiempos de estudio: T0 antes del inicio de inmunoterapia, T1 al alcanzar el mantenimiento, T2 a los 6 meses de éste y T3 al año del mantenimiento.

La monitorización de la eficacia del tratamiento se realizó en base a una valoración clínica (cartilla de síntomas y medicación, registro de pico de flujo, cuestionario de calidad de vida ${ }^{22,23}$, evaluación de la enfermedad y del tratamiento por parte del paciente y del médico y valoración de la severidad de la enfermedad por el médi$\left.\operatorname{co}^{24,25}\right)$, análisis de parámetros "in vivo" (pruebas cutáneas en prick ${ }^{21}$ y provocación conjuntival ${ }^{26}$ ) e "in vitro" (IgE e IgG específicas anti- $A$. alternata) y monitorización de la presión alergénica externa ${ }^{27}$ e interna ${ }^{28}$.

La seguridad del tratamiento se monitorizó de modo continuo mediante el registro de todas las reacciones adversas, tanto locales como sistémicas, inmediatas y tardías, según criterios de la EAACI ${ }^{10}$.

Resultados: el 70\% de los pacientes seleccionados fueron varones y la edad media fue de 13,89 años. El 90\% padecían asma, siendo la media de la severidad de la rinitis de 7,46 (escala de 0 a 14) y del asma de 4,56 (escala de 0 a 13). La mitad de los pacientes fueron asignados al grupo placebo y la otra mitad al activo.

Cinco pacientes no finalizaron el estudio: dos de ellos fueron retirados por inestabilidad de su enfermedad de base y los otros 3 lo abandonaron por propia iniciativa por diferentes motivos personales.

Todos los pacientes alcanzaron la dosis preestablecida de mantenimiento de 1670 UBE.

En el grupo activo encontramos mejoría en los síntomas pulmonares y en el score combinado de síntomas y medicación en T2 respecto a $\mathrm{T} 0$. El registro del pico de flujo (Fig. 3), la evaluación de la enfermedad por el paciente (Fig. 4) y del tratamiento por el médico y la severidad de la rinitis y del 
asma (Fig. 5) mejoraron en todos los tiempos de estudio analizados. La provocación conjuntival mejoró en $\mathrm{T} 1$ respecto a $\mathrm{T} 0$. Los síntomas oculares y nasales, así como los pulmonares en el resto de tiempos de estudio, los cuestionarios de calidad de vida y la reactividad conjuntival en el resto de tiempos mostraron una tendencia a la mejoría sin llegar a alcanzar una significación estadística. No hubo resultados concluyentes en los test cutáneos. Respecto a los parámetros "in vitro" la IgG sérica específica aumentó y la IgE sérica específica disminuyó en todos los tiempos de estudio respecto a T0 (Fig. 6)

En el grupo placebo se produjo una mejoría en T3 en el score combinado de síntomas y medicación, en la valoración de la enfermedad por el paciente (Fig. 4) y en la severidad de la rinitis (Fig. 5). Por el contrario, el área bajo la curva del prick experimentó un aumento al final del estudio respecto al inicio. No hubo cambios significativos en el resto de parámetros analizados.

Únicamente se han registrado 2 reacciones sistémicas leves (una RSI-1 y otra RST-RC) en las 711 dosis administradas $(0,28 \%$ del total de dosis).

\section{CONCLUSIONES}

Se ha obtenido y evaluado un extracto de Alternaria alternata de alta calidad para el diagnóstico y tratamiento de pacientes alérgicos a este hongo.

Se ha estimado la dosis máxima tolerada para inmunoterapia con dicho extracto en una preparación depot y mediante una pauta convencional, en $0,1 \mathrm{mg} / \mathrm{ml}$ de Alt a

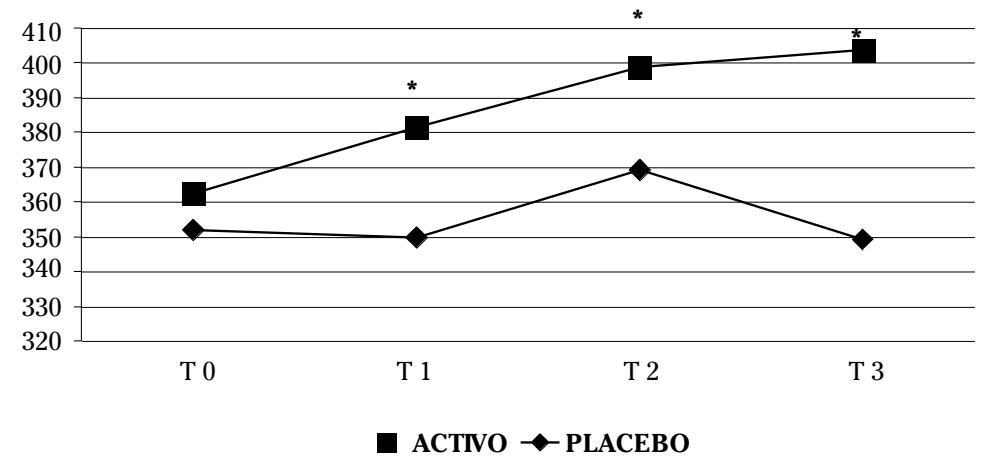

Figura 3. Registro de pico de flujo (PEF).

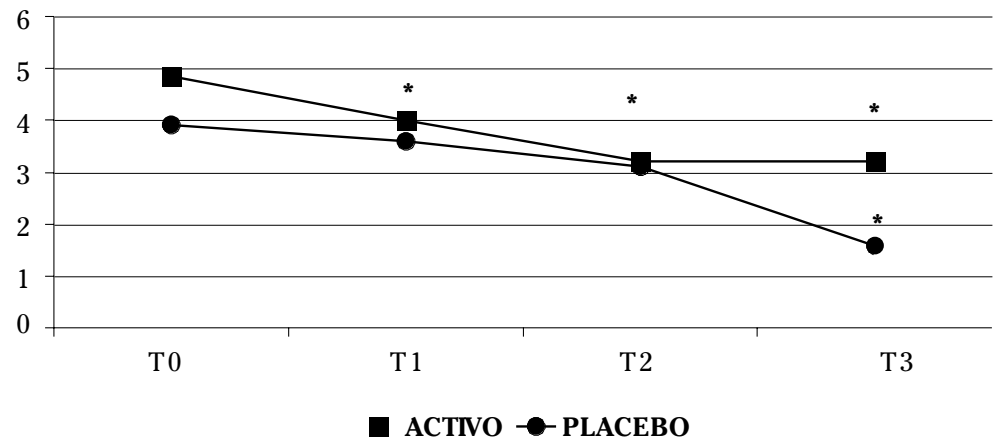

Figura 4. Evaluación de la enfermedad por los pacientes. 


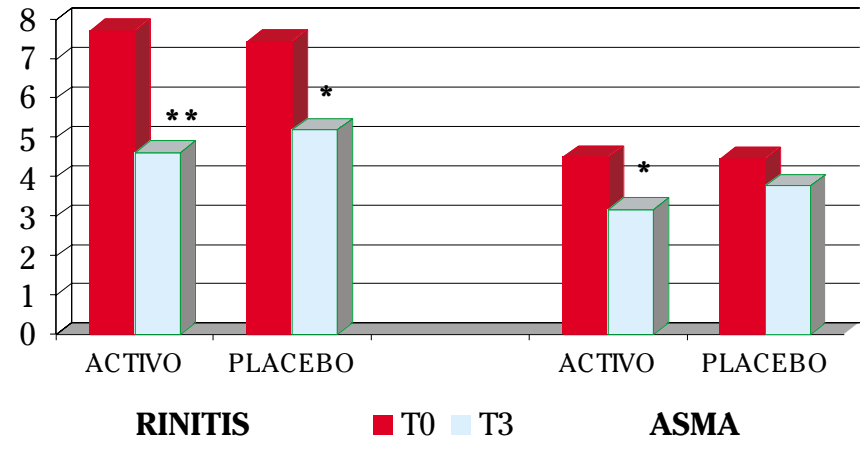

Figura 5. Severidad de la enfermedad.

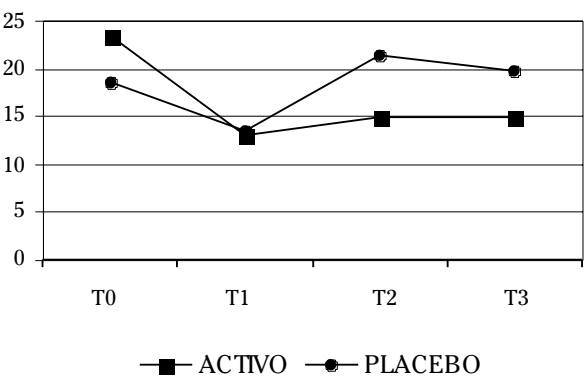

IgE

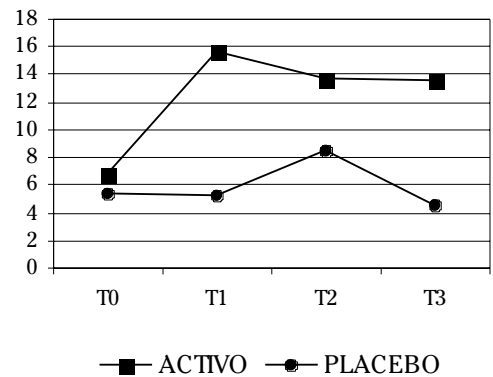

IgG

Figura 6. Determinación de IgE e IgG específicas anti-Alternaria.

1. Esta dosis ha sido establecida como la dosis de mantenimiento de inmunoterapia en un ensayo doble ciego realizado para evaluar la eficacia y seguridad del tratamiento.

La inmunoterapia con este extracto es efectiva al mejorar los síntomas de los pacientes.

Los parámetros "in vivo" evaluados muestran una tendencia al cambio en el grupo activo.

Los datos obtenidos en los test "in vivo" confirman una respuesta inmunológica adecuada a la inmunoterapia.

La tolerancia del tratamiento ha sido excelente, registrándose únicamente dos reacciones leves en las 711 dosis administradas $(0,28 \%$ del total de dosis $)$.

\section{BIBLIOGRAFÍA}

1. D’Amato G, Chatzigeorgiou G, Corsico R GIOULEKAS D, JÄGER L, JÄGER S, et al. Evaluation of the prevalence of skin prick test positivity to Alternaria and Cladosporium in patients with suspected respiratory allergy. EEACl Position Paper. Allergy 1997; 52: 711-716.

2. D’Amato G, Spieksma FTM. Aerobiologic and clinical aspects of mould allergy in Europe. EAACI Position Paper. Allergy 1995; 50:870 877.

3. SAlvaggio J, Aukrust L. Mold-induced asthma J Allergy Clin Immunol 1981; 68: 327-346.

4. Pérez Santos C, Moreno AG. En: Hongos y alergia. Madrid: Dome/Hollister-Stier. 1992: 93.

5. SLY RM. New guidelines for diagnosis and management of asthma. Ann Allergy Asthma Immunol 1997; 78: 427-437. 
6. Van Caumenberge P, Bachert C, Passalacqua G, Bousquet J, CANONiCa GW, Durham SR et al. Consensus statement on the treatment of allergic rhinitis. Allergy 2000; 55: 116-134.

7. Bousquet J, LOCKEY RF, Malling HG. WHO Position Paper. Allergen Immunotherapy: therapeutic vaccines for allergic diseases. Allergy 1998; $53: 1-42$.

8. Abramson MJ, Puy RM, WeinER JM. Is allergen immunotherapy effective in asthma? Am J Respir Crit Care Med 1995; 151: 969-975.

9. ABramson MJ, PuY RM, WEINER JM. Immunotherapy in asthma: an updated systematic reviw. Allergy 1999; 54: 1022-1041.

10. MALLING HJ, WeEKE B. EAACI Immunotherapy Subcommittee. Position Paper: Immunotherapy. Allergy 1993; 48: 1-45.

11. KAAD PH, OSTERGAARD PA. The hazard of mould hyposensitization in children with asthma. Clin Allergy 1982; 12: 317-320.

12. OstergaArd PA, KaAd PH, KRISTENSEN T. A prospective study on the safety of immunotherapy in children with severe asthma. Allergy 1986; 41: 588-593.

13. Dreborg S, Agrell B, Foucard T, KJellman M, KOIVIKKO A, NILSSON S. A double-blind, multicenter immunotherapy trial in children, using a purified and standardized Cladospurium herbarum preparation. I. Clinical results. Allergy 1986, 41: 131-140.

14. Martorell A, Sole A, Diez LV, Belenguer J, Sanz $\mathrm{J}$, TORRo MI et al. Inmunoterapia en alergia a hongos. Rev Esp Alergol Inmunol Clin 1986; 1: 188-192.

15. Malling HJ, Dreborg S, Weeke B. Diagnosis and immunotherapy of mould allergy. V. Clinical efficacy and side effects of immunotherapy with Cladosporium herbarum. Allergy 1986; 41: 507-519.

16. CANTANi A, Businco E, Maglio A. Alternaria allergy: A three-year controlled study in children treated with immunotherapy. Allergol et immunopathol 1988; 16: 1-4

17. Horst M, Hejuadi A, Horst V, Michel B Bousouet J. Double-blind, placebo-controlled rush immunotherapy with a standardized Alternaria extract. J Allergy Clin Immunol 1990; 85: 460-72

18. Tabar AI, Lizaso MT, García BE, Echechipía $S$, Olaguibel JM, RodRíGuez A. Tolerance of immunotherapy with a standardized extract of Alternaria tenuis in patients with rhinitis and bronchial astma. J Investig Allergol Clin Immunol 2000; 10: 327-333.

19. LOMBARdERo M, GonZÁlez R, DuFfort O, JuAN F, Ayuso R, Ventas $\mathrm{P}$ et al. Evaluación de la actividad biológica total y composición alergénica de extrtactos alergénicos. Allergol Immunopathol19986; 14: 189-198.

20. TuRKeltaub PC. Biological standardization of allergenic extracts. Allergol Immunopatho 1989; 17: 53-65.

21. EAACI Subcommittee on Skin tests. Allergen standardization and skin tests. Position Paper. Allergy 1993; 48: 48-82.

22. Juniper EF, GuYATt GH, EPSTEIN RS, FERrie PJ, JAESCHKE R, HILLER TK. Evaluation of impairment of health related quality of life in asthma: development of a questionnaire for use in clinical trials. Thorax 1992; 47: 76-83.

23. Sanjuas C, Alonso J, SAnchis J, Casan P Broquetas JM, Ferrie PJ et al. The quality of life questionnaire with asthma patients; the Spanish version of the Asthma Quality of Life Questionnaire. Archiv Bonconeumol 1995; 31: 219-226.

24. AAS K. Heterogeneity of bronchial asthma Allergy 1981; 36: 3-14.

25. MELTZER EO. Evaluating rhinitis: clinical, rhinomanometric and cytologic assessments. J Allergy Clin Immunol 1988; 82: $900-908$.

26. MÖLlER CH, BJÖRKSTEN B, BNILSSON G, DREBORG S. The precision of the bronchial provocation test. Allergy 1984; 39: 37-41.

27. HiRST JM. An automatic volometric spore trap. Ann Appl Biol 1952; 39: 257-265.

28. CHAPMAN MD. Environmental allergen monitoring and control. Allergy 1998; 53: 4853. 